\title{
Variable liquid crystal pretilt angles generated by photoalignment of a mixed polyimide alignment layer
}

\author{
Jacob Y. L. Ho, ${ }^{\text {a) }}$ V. G. Chigrinov, and H. S. Kwok \\ Center for Display Research, Hong Kong University of Science and Technology, \\ Clear Water Bay, Hong Kong
}

(Received 2 February 2007; accepted 19 May 2007; published online 13 June 2007)

\begin{abstract}
A method to generate any liquid crystal pretilt angle is proposed and demonstrated. This method is based on deep ultraviolet photoalignment of a layer consisting of nanostructured domains of horizontal and vertical alignment polyimides. Competition between the two types of domains produces the controlled pretilt angle. The obtained anchoring energy is reasonably large. (C) 2007 American Institute of Physics. [DOI: 10.1063/1.2748345]
\end{abstract}

Materials such as polyimide (PI), photopolymers, ${ }^{1,2}$ surfactants, ${ }^{3}$ and silicon oxide ${ }^{4}$ have been used to align liquid crystals (LCs). Among them, PI is the most popular because of its excellent thermal stability, chemical resistance, high mechanical strength, good transparency, adhesive strength, and strong anchoring energies. To align LC on PI, methods such as mechanical rubbing, ion beam irradiation, ${ }^{5,6}$ plasma treatment, ${ }^{7}$ and polarized deep ultraviolet (DUV) light illumination have been used. The conventional rubbing process, though popular in mass production of LC displays, can only generate pretilt angles of less than $10^{\circ}$ from the horizontal or vertical direction. This precludes applications such as bistable bend-splay displays, ${ }^{8}$ no-bias bend displays, ${ }^{9}$ and for the production of O-plate compensation films.

Recently, much effort has been devoted to the development of photoalignment of LC. Photoalignment is a noncontact method that does not suffer from the drawbacks of mechanical rubbing. ${ }^{10-16}$ DUV radiation of PI is one of the photoalignment methods explored. It retains the good thermal stability of the material since the radiation is performed after the imidization process. However, most studies on DUV radiation of PI have so far can generate pretilt angles of less than $3^{\circ} .^{14,15}$ Nishikawa et al. showed that pretilt angles up to $30^{\circ}$ can be achieved by changing the mole fraction of $\mathrm{F}$ diamine in PI. ${ }^{12}$ For pretilt angles from $10^{\circ}$ to $30^{\circ}$, large uncertainty exists even for the same mole fraction of $\mathrm{F}$ diamine, so that large pretilt angles are difficult to achieve reproducibly.

In a previous study, we used a mixture of horizontal and vertical alignment PIs to produce arbitrary pretilt angles by nanostructure formation. ${ }^{17}$ Mechanical rubbing was required. Here, we show that photoalignment can be used to achieve the same results. We report a simple and controllable method for producing a wide range of pretilt angles $\left(20^{\circ}-90^{\circ}\right)$ of $\mathrm{LC}$ by photoalignment of a mixed PI alignment layer. Alignment of LC in the azimuthal direction is produced by in situ DUV photoalignment.

The materials used in the present study were homogenous $(H)$ polyimide from Chisso Corporation and homeotropic $(V)$ polyimide from the Japan Synthetic Rubber Company. Different concentrations of homeotropic PI were prepared by mixing the $H$ - and $V$-polyimides accordingly. The PI film was formed on an indium tin oxide glass sub-

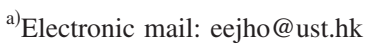

strate by spin coating and baking at $100{ }^{\circ} \mathrm{C}$ for $5 \mathrm{~min}$. The coated substrate was then put on a hot plate $\left(220^{\circ} \mathrm{C}\right)$ under a vacuum for imidization. A nitrogen environment can also be used to prevent oxidation of the PI during the imidization process. During the imidization process, polarized DUV light was used to expose the PI, inducing anisotropy in the azimuthal direction in the alignment layer. It was then followed by unpolarized DUV light exposure at an oblique angle to produce anisotropy in the polar direction.

Figure 1 shows a schematic diagram of the two-step DUV exposure in situ imidization setup. A collimated beam of DUV light from a $1000 \mathrm{~W} \mathrm{Hg-Xe} \mathrm{lamp} \mathrm{was} \mathrm{polarized} \mathrm{by}$ a Glan-Taylor polarizer. The intensity of the DUV beam was $14 \mathrm{~mW} / \mathrm{cm}^{2}$. The PI-coated substrate was illuminated at normal incidence by the polarized beam at a dosage of $7 \mathrm{~J} / \mathrm{cm}^{2}$. Then, the substrate was illuminated by oblique unpolarized DUV light $\left(40 \mathrm{~mW} / \mathrm{cm}^{2}\right)$ at an incident angle of $30^{\circ}$ at a dosage of $1.2 \mathrm{~J} / \mathrm{cm}^{2}$. During the entire photoalignment process, the substrate was placed under a vacuum and was kept at a constant temperature of $220^{\circ} \mathrm{C}$.

After photoalignment, antiparallel liquid crystal cells with $3.2 \mu \mathrm{m}$ cell gaps were assembled. The cells were filled with MLC-6235-000 liquid crystals from Merck. The pretilt angle of the LC cell was measured by the crystal rotation method. ${ }^{18}$ The pretilt angle as a function of the concentration of the homeotropic PI is shown in Fig. 2. The graph shows that by varying the concentration from 30 to $60 \mathrm{wt} \%$, a wide range of pretilt angles can be generated. Also, the pretilt angle is almost linearly dependent on the $V$-PI concentration and the slope is approximately $2 \% / \mathrm{wt} \%$. Therefore, we can tune the pretilt angle accurately and easily with a very wide process window. Figure 3 shows photographs of antiparallel cells under crossed polarizers. From the uniform color of the images of different cells, it can be seen that the pretilt angle is very uniform.

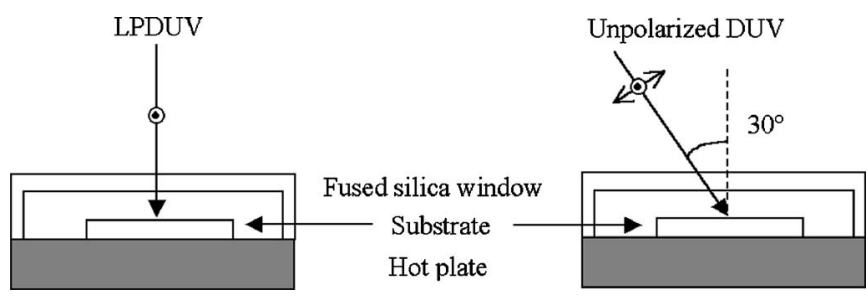

FIG. 1. Schematic diagrams of the in situ imidization process and the twostep DUV exposure setup. 


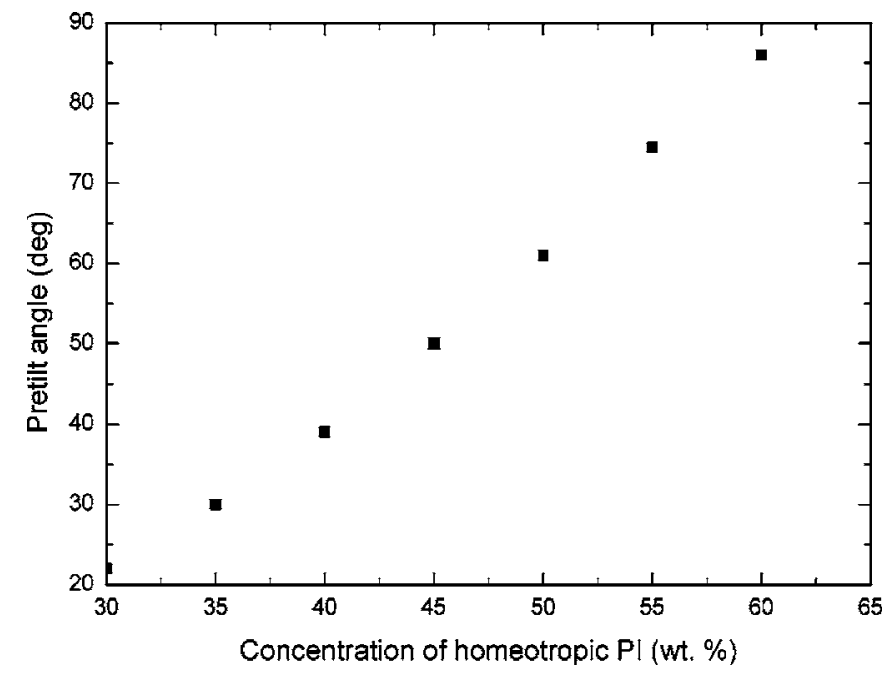

FIG. 2. Measured pretilt angle as a function of the concentration of homeotropic PI.

The pretilt angle actually depends on quite a number of parameters, namely, the surface area ratio of the two types of PI, the anchoring strength, the relative drying and polymerization rate of the PIs, and the liquid crystal. The illumination intensity and duration also play some role because they affect the chemical bond dissociation process. It should also be noted that the surface area ratio is not necessarily the same as the volume ratio of the PI due to differential precipitation. For example, if all the $H$-PI precipitates before the $V$-PI does, then it is possible that the surface will be covered with $V$-PI no matter what the original volume ratio was in the liquid form. This is the reason why, when the concentration of $V$-PI is above $60 \mathrm{wt} \%$, the pretilt angle approaches $90^{\circ}$. To obtain a wide process window, the anchoring strength of both types of PI should be nearly the same. The imidization temperature should be chosen such that the time is long enough for polymerization of both polyimides.

It is believed that the mechanism of photoalignment by DUV light is via bond breaking in the preferred direction. The first irradiation step produces a preferred direction for both types of polyimides on the alignment surface, either parallel or perpendicular to the polarization of the incident light. The second irradiation step with unpolarized DUV light at an angle breaks the symmetry to get a preferred tilt-

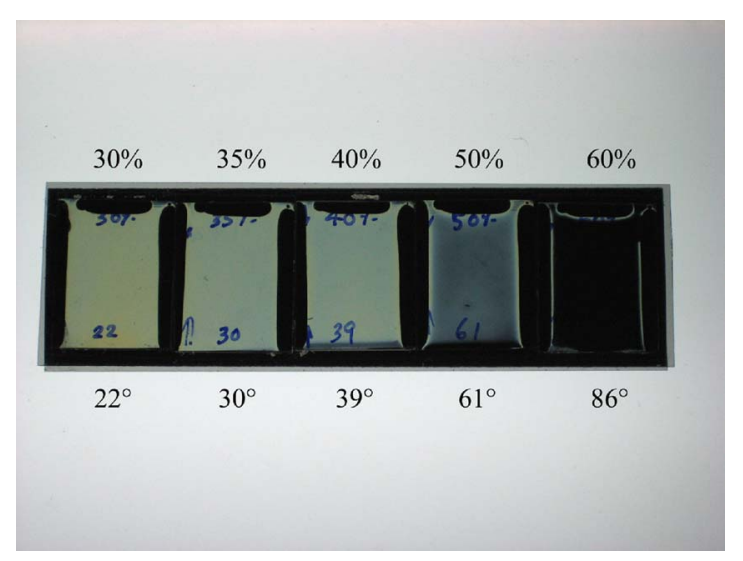

FIG. 3. (Color online) Photographs of antiparallel cells under crossed polarizers. The numbers written above the samples indicate the concentration of the homeotropic PI and the numbers below the sample indicate the pretilt

angle.
Downloaded 08 Sep 2008 to 143.89.106.152. Redistribution subject to AIP license or copyright; see http://apl.aip.org/apl/copyright.jsp
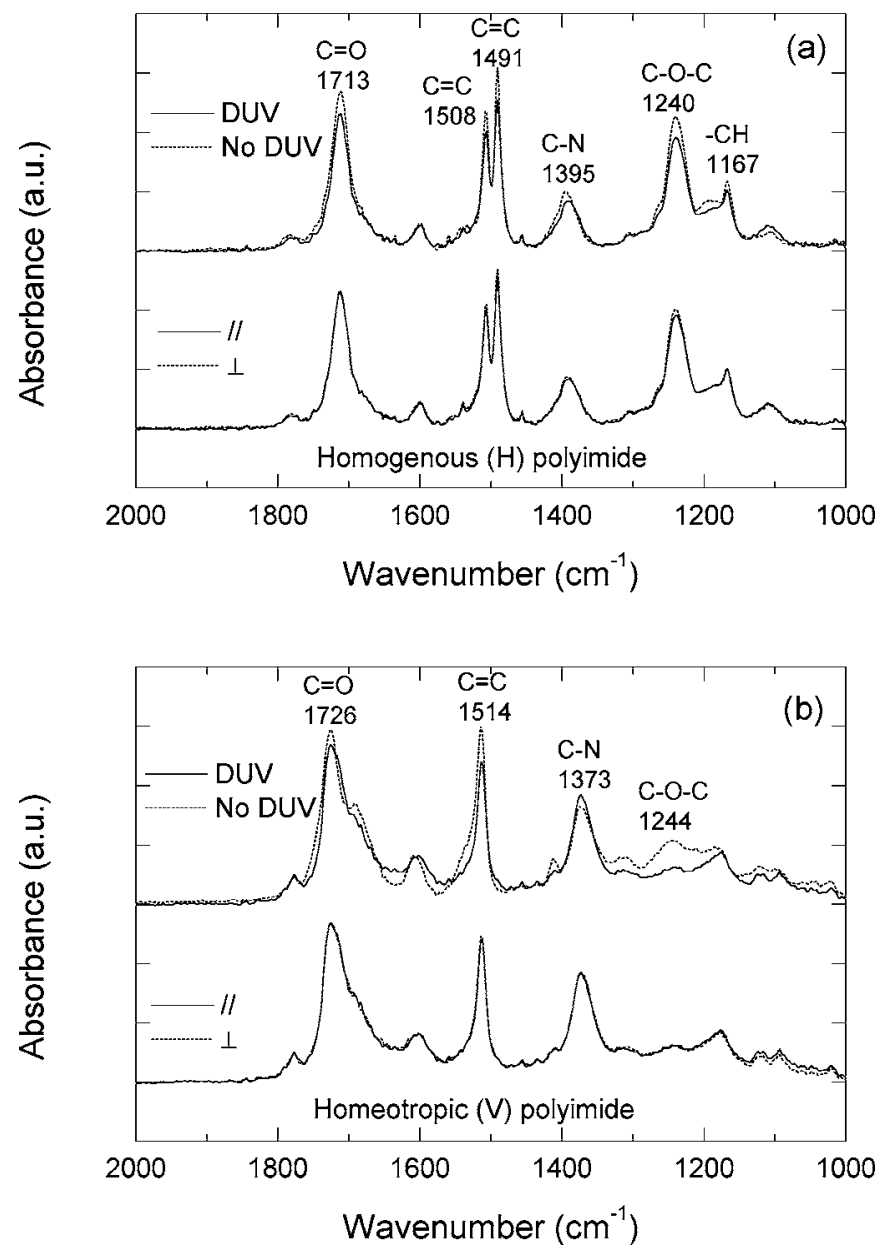

FIG. 4. FTIR spectra of (a) homogenous PI and (b) homeotropic PI. The upper two curves of both figures show the infrared spectra before and after DUV illumination. The lower two curves show the dichroic infrared spectra when the polarization direction of infrared is parallel and perpendicular to the polarization direction of the DUV light.

ing direction for the alignment layer. To further investigate the mechanism of alignment of $H$ - and $V$-polyimides caused by DUV exposure, we performed Fourier transform infrared (FTIR) spectroscopy to obtain structural information on physicochemical changes of the polyimides upon radiation. The measurements were performed with a BioRad FTS6000 spectrometer and the polyimide films were coated on silicon wafers. Figure 4(a) shows the FTIR spectra of the homogenous PI. The characteristic absorption peaks are associated with the $\mathrm{C}=\mathrm{O}$ stretching mode $\left(1713 \mathrm{~cm}^{-1}\right)$ and the $\mathrm{C}-\mathrm{N}$ stretching mode $\left(1395 \mathrm{~cm}^{-1}\right)$ of the imide group, the $\mathrm{C}=\mathrm{C}$ stretching mode $\left(1508\right.$ and $\left.1491 \mathrm{~cm}^{-1}\right)$ of the aromatic ring, the $\mathrm{C}-\mathrm{O}-\mathrm{C}$ stretching mode $\left(1240 \mathrm{~cm}^{-1}\right)$, and the $-\mathrm{CH}$ stretching mode $\left(1167 \mathrm{~cm}^{-1}\right)$. The upper curves show that the intensities of all of the peaks decrease after DUV illumination. These results indicate that bond-breaking reactions occur in the homogenous PI. The lower two curves show the FTIR dichroic spectra when the polarization direction of the infrared was parallel and perpendicular to the polarization direction of the DUV light. The intensities of the absorption peaks at 1508,1491 , and $1240 \mathrm{~cm}^{-1}$ were lower when the polarization direction of the infrared light was parallel to the polarization direction of the DUV light. This indicates that the $\mathrm{C}=\mathrm{C}$ and $\mathrm{C}-\mathrm{O}-\mathrm{C}$ bonds dissociate faster along the polarized DUV light direction. In other words, the number of lo AIP license or copyright; see http://apl.aip.org/apl/copyright.jsp 


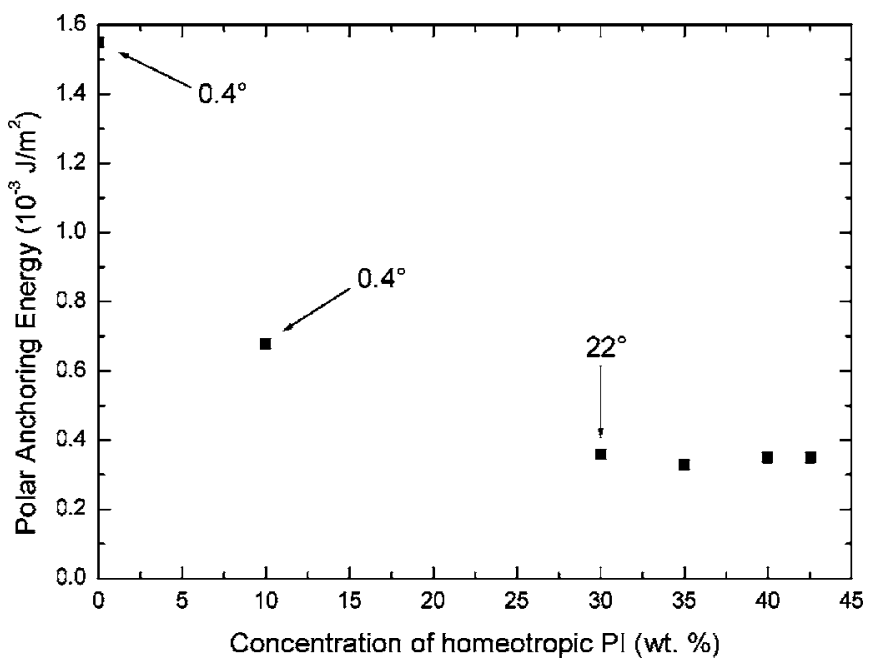

FIG. 5. Dependence of the polar anchoring energy on the concentration of homeotropic PI.

polymer main chains is higher in the direction perpendicular to the polarized DUV light direction, causing a preferential alignment of the liquid crystal molecules perpendicular to the polarized light direction.

The FTIR spectra of the homeotropic PI are shown in Fig. 4(b). Similar bond-breaking processes are observed in the upper curves except the fact that the intensity of the absorption peak at $1373 \mathrm{~cm}^{-1}$ increases after the DUV exposure. This may be due to the oxidation of this particular polymer fragment during the bond-breaking process by the residual oxygen in the vacuum at high temperature. On the other hand, the dichroic spectra of the lower two curves show no observable anisotropy. This means that the in-plane alignment of the LC is mainly derived from the homogenous PI rather than from the homeotropic PI. The main function of the homeotropic PI is the out-of-plane alignment of the LC.

For the DUV alignment method to be useful, the polar anchoring strength of the LC alignment layer should not be too weak. We measured the polar anchoring energy (W) of the LC cells by the high-field technique (referred to as the retardation versus voltage technique), which is based on the measurement of retardation versus applied voltage. ${ }^{19}$ Figure 5 plots the measured polar anchoring energy in relation to the concentration of homeotropic PI. As can be seen, $\mathrm{W}$ decreases from its maximum value and approaches a constant value as the concentration of $V$-PI increases. This is due to the fact that the anchoring strength of $H$-PI is stronger than that of $V$-PI. When the concentration of $V$-PI is low, the effect of $H$-PI is dominant. Therefore, the value of $\mathrm{W}$ ranges from $1.55 \times 10^{-3}$ to $0.68 \times 10^{-3} \mathrm{~J} / \mathrm{m}^{2}$ for pure $H$-PI and 10 wt $\% V$-PI, respectively. On the contrary, when the concentration of $V$-PI is larger than $30 \mathrm{wt} \%$, the effect of $V$-PI is dominant. This explains why $\mathrm{W}$ approaches a constant value. Stronger values of $\mathrm{W}$ with large pretilt angles can be achieved by using a $V$-PI with a stronger anchoring strength. We note that the value of $\mathrm{W}$ of $H$-PI generated by DUV is comparable to conventional values produced by rubbing. Moreover, the range of $\mathrm{W}$ can be tuned without altering the pretilt angle by varying the concentration of $V$-PI from 0 to say $15 \mathrm{wt} \%$. This is useful especially in bistable displays, which require weak anchoring alignment layers. ${ }^{20}$

In conclusion, we have demonstrated a method for generating a wide range of liquid crystal pretilt angles. This method has the advantages of simplicity, a wide process window, and a low exposure dose of DUV light. It is also a nonrubbing method and is based on photoalignment. The process time is short because the baking process is carried out in situ with the DUV alignment process.

This research was supported by the Hong Kong Government Innovation and Technology Fund.

${ }^{1}$ M. Schadt, K. Schmitt, V. Kozenkov, and V. Chigrinov, Jpn. J. Appl. Phys., Part 1 31, 2155 (1992).

${ }^{2}$ T. Hashimoto, T. Sugiyama, K. Katoh, T. Saitoh, H. Suzuki, Y. Limura, and S. Kobayashi, SID Int. Symp. Digest Tech. Papers 26, 877 (1995).

${ }^{3}$ K. Ogawa, T. Ohtake, and T. Nomura, Jpn. J. Appl. Phys., Part 1 41, 6471 (2002).

${ }^{4}$ J. L. Janning, Appl. Phys. Lett. 21, 173 (1972).

${ }^{5}$ P. Chaudhari, J. A. Lacey, S. C. A. Lien, and J. L. Speidell, Jpn. J. Appl. Phys., Part 2 37, L55 (1998).

${ }^{6}$ J. Stöhr, M. G. Samant, J. Lüning, A. C. Callegari, P. Chaudhari, J. P. Doyle, J. A. Lacey, S. A. Lien, S. Purushothaman, and J. L. Speidell, Science 292, 2299 (2001).

${ }^{7}$ O. Yaroshchuk, R. Kravchuk, A. Dobrovolskyy, L. Qiu, and O. D. Lavrentovich, Liq. Cryst. 31, 859 (2004).

${ }^{8}$ X. J. Yu and H. S. Kwok, SID Int. Symp. Digest Tech. Papers 35, 875 (2004).

${ }^{9}$ F. S. Y. Yeung and H. S. Kwok, Appl. Phys. Lett. 88, 063505 (2006).

${ }^{10}$ J. Chen, D. L. Johnson, P. J. Bos, X. Wang, and J. L. West, Phys. Rev. E 54, 1599 (1996).

${ }^{11}$ J. H. Kim, S. Kumar, and S. D. Lee, Phys. Rev. E 57, 5644 (1998).

${ }^{12}$ M. Nishikawa, B. Taheri, and J. L. West, SID Int. Symp. Digest Tech. Papers 29, 131 (1998).

${ }^{13}$ J. H. Kim, B. R. Acharya, S. Kumar, and K. R. Ha, Appl. Phys. Lett. 73, 3372 (1998).

${ }^{14}$ D. S. Seo, and H. K. Kim, Jpn. J. Appl. Phys., Part 2 39, L993 (2000).

${ }^{15}$ J. H. Kim, B. R. Acharya, D. M. Agra, and S. Kumar, Jpn. J. Appl. Phys., Part 1 40, 2381 (2001).

${ }^{16}$ J. Y. Hwang and D. S. Seo, Jpn. J. Appl. Phys., Part 1 40, 4160 (2001).

${ }^{17}$ F. S. Yeung, J. Y. Ho, Y. W. Li, F. C. Xie, O. K. Tsui, P. Sheng, and H. S. Kwok, Appl. Phys. Lett. 88, 051910 (2006).

${ }^{18}$ J. S. Gwag, S. H. Lee, K. H. Park, W. S. Park, K. Y. Han, C. G. Jhun, T. H. Yoon, J. C. Kim, D. M. Song, and D. M. Shin, J. Appl. Phys. 93, 4936 (2003).

${ }^{19}$ Y. A. Nastishin, R. D. Polak, S. V. Shiyanovskii, V. H. Bodnar, and O. D. Lavrentovich, J. Appl. Phys. 86, 4199 (1999).

${ }^{20}$ I. Dozov, P. M. Lagarde, S. L. Forget, D. Stoenescu, J. Angelé, R. Vercelletto, B. Pécout, and A. Boissier, SID Int. Symp. Digest Tech. Papers 32, 224 (2001). 